\title{
Hydroxyzine revealing acquired neuromuscular weakness in a patient with COVID-19 disease
}

\author{
Mohamed Bahi MD, Younes Aissaoui MD, Ayoub Belhadj MD, Youssef Qamouss MD, Rachid Seddiki MD
}

\begin{abstract}
Acquired neuromuscular weakness often develops in patients with an acute respiratory distress syndrome (ARDS), in particular in patients who are ventilated and sedated for long periods. This has been rarely described in the literature on ARDS secondary to SARS-CoV-2 infection. Our clinical case revealed the existence of these neuromuscular manifestations in the COVID-19 disease after the use of hydroxyzine, an antihistamine whose respiratory sideeffects are unknown.
\end{abstract}

Keywords: Neuromuscular weakness, COVID-19, hydroxyzine, hypercapnia.

\section{INTRODUCTION}

The ongoing COVID-19 pandemic has affected many patients by causing severe respiratory infection. However, the literature regarding neuromuscular complications with these infections is scant. This clinical case highlights these complications, in particular acquired neuromuscular weakness in intensive care, in a case in which the diagnosis was triggered by hydroxyzine, a drug with rare respiratory side-effects.

\section{Clinical case}

A 59-year-old patient with no medical history was admitted with acute respiratory distress. These symptoms started five days prior to presentation with the appearance of fever, generalized arthralgias and myalgias, and cough. Given the clinical probability of SARS-CoV2-severe interstitial lung disease, viral testing by RT-PCR on a nasopharyngeal swab was performed and was positive. Thoracic $\mathrm{X}$-ray revealed a bilateral interstitial syndrome (Figure 1).

Corresponding author: Mohamed Bahi

Contact Information: Bahi.mohamed11@gmail.com

DOI: $10.12746 /$ swrccc.v9i40.875
Thoracic computed tomography (CT) scan documented ground glass opacities with crazy paving classified as CO-RADS 5 (Figure 2).

The initial treatment consisted of a high concentration oxygen therapy by mask with a $92 \%$ oxygen saturation target and a chloroquine and azithromycin protocol (500 mg of chloroquine twice a day for 10 days

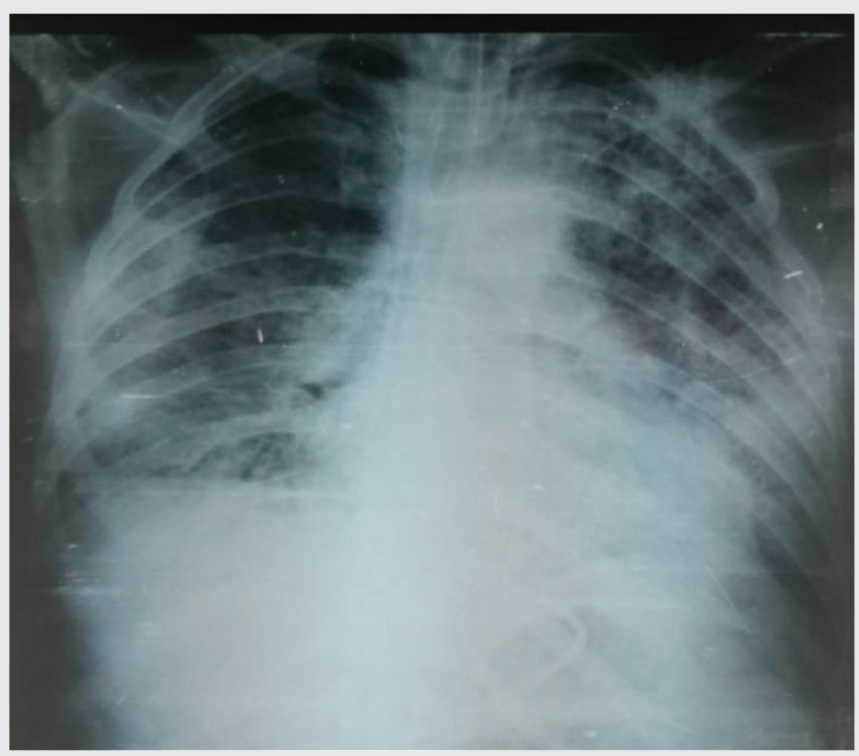

Figure 1. Bilateral interstitial syndrome. 


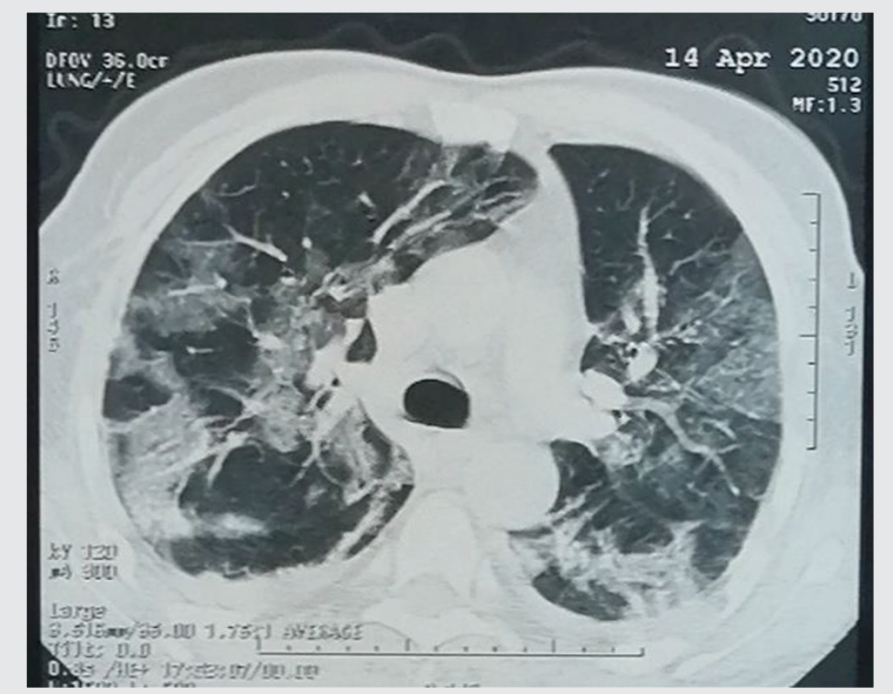

Figure 2. Thoracic CT scan with ground glass opacities and crazy paving classified as CO-RADS 5.

and azithromycin $500 \mathrm{mg}$ on Day1 then $250 \mathrm{mg}$ from Day 2 to Day 7). His course was marked by increasing symptoms despite the use of noninvasive ventilation with significant hypoxemia on arterial blood gases (ABG) $\left(\mathrm{PaO}_{2}\right.$ at $45 \mathrm{mmHg}$ with $\mathrm{PaO}_{2} / \mathrm{FiO}_{2}$ at 75$)$ and the extension of ground glass areas with posterior and basal condensations on CT scan (Figure 3).

Given this course, the patient was intubated, ventilated, and sedated on day 12 of his admission with continuous rocuronium paralysis for the first 24 hours and administration of dexamethasone $6 \mathrm{mg}$ per day for 10 days. After 5 sessions of 18 hours per day prone positioning, there was improvement in hypoxemia $\left(\mathrm{PaO}_{2}\right.$ from 77 to $87 \mathrm{mmHg}$ with $\mathrm{PaO}_{2} / \mathrm{FiO}_{2}$ from 128 to 217). Follow-up thoracic CT noted stabilization of ground glass images with regression of the initial condensations.

The patient was extubated on day 17 . He remained tachypneic with a respiratory rate of 38 breaths per minute on $10 \mathrm{~L} / \mathrm{min}$ of oxygen. On day 21 , hydroxyzine $1 \mathrm{mg} / \mathrm{kg} / \mathrm{day}$ was administered due to the persistence of nocturnal insomnia. On day 23 , his course was marked by the occurrence of hypercapnia at $\mathrm{PaCO}_{2} 77 \mathrm{mmHg}$ and then at $90 \mathrm{mmHg}$ and paradoxically a respiratory improvement with a decrease in

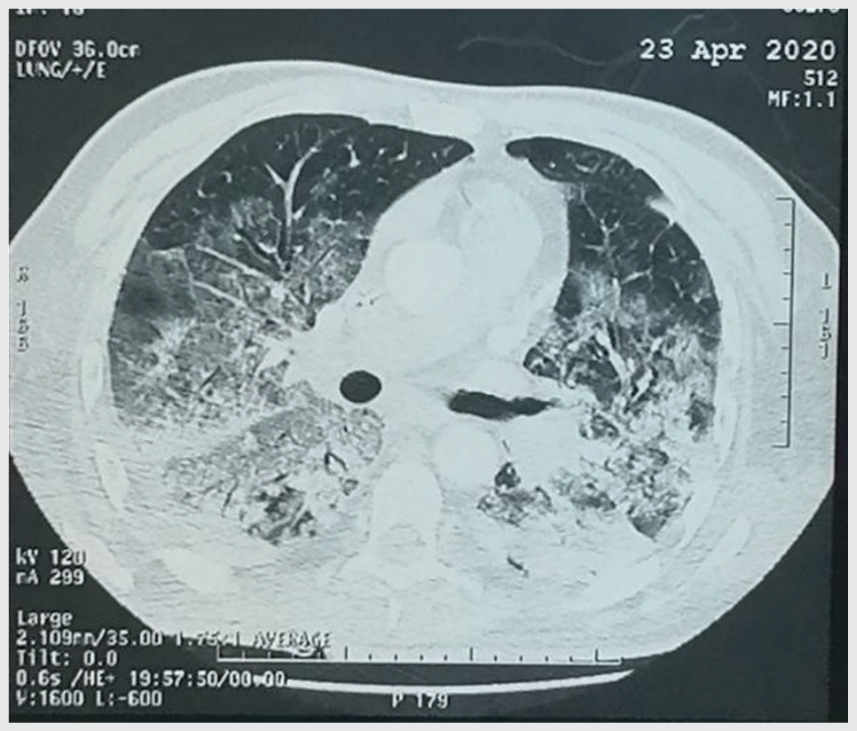

Figure 3. Thoracic CT scan with extension of ground glass areas with posterior and basal condensations.

respiratory rate to 25 breaths per minute and a $\mathrm{PaO}_{2}$ at $91 \mathrm{mmHg}$ with $\mathrm{PaO}_{2} / \mathrm{FiO}_{2}$ at 225 . The patient had daytime sleepiness with fatigue and heavy sweating. An interruption of the administration of hydroxyzine was decided.

Given this hypercapnic respiratory failure, an electroneuromyographic (ENMG) study was performed to evaluate him for acquired neuromuscular weakness and demonstrated an ENMG pattern of motor axonal neuropathy with diaphragmatic involvement. Spontaneous evolution was marked by a decrease in hypercapnia to 69,62 , and then $52 \mathrm{mmHg}$ on ABG and gradual withdrawal from oxygen with respiratory improvement. After an early period of rehabilitation and locomotor physiotherapy, the patient was discharged on day 35 with home oxygen therapy.

\section{Discussion}

\section{COVID-19 AND NEUROMUSCULAR DISORDERS}

Growing evidence suggests that the SARS-CoV-2 virus has neuroinvasive potential, like other coronaviruses. One study from COVID-19 designated hospitals in Wuhan, China, demonstrated that more than 
one-third of Coronavirus patients presented with neuromuscular syndromes, including evidence of skeletal muscle damage. ${ }^{1}$ A systematic literature search from December 01, 2019, to May 14, 2020, identified 82 cases of COVID-19 with neurological complications, and $28 \%$ had neuromuscular disorders (NMD). ${ }^{2}$

\section{RISK OF TREATMENTS FOR NEUROMUSCULAR DISORDERS ASSOCIATED WITH COVID-19}

Prolonged use of non-depolarizing paralytics is strongly associated with the development of acquired muscular weaknesses in intensive care. Indeed, weakness related to type 2 muscle fiber atrophy from disuse typically presents after 1 week in ill patients who are bedridden and therefore will be a consideration in patients with COVID-19.,

Chloroquine and hydroxychloroquine have also been mentioned as possible treatment for COVD-19. They have also been associated with new-onset or worsening myasthenia gravis and are typically used with caution in this patient group. ${ }^{5,6}$ Azithromycin, a macrolide antibiotic used with chloroquine or hydroxychloroquine for COVID-19, may also cause worsening of myasthenia gravis. ${ }^{7}$

\section{RESPIRATORY EFFECTS OF HYDROXYZINE}

Hydroxyzine hydrochloride is a minor tranquilizer that is widely used as a premedication for sedation to reduce preoperative anxiety without respiratory depression. However, a few studies have shown a slight but significant respiratory depressant effect of hydroxyzine given intramuscularly. Lauria et al. studied the respiratory effects of hydroxyzine and concluded that the response to elevated endogenous carbon dioxide was variable and inconsistent, ${ }^{8}$ a fact that we noticed in our patient who developed significant hypercapnia, due mainly to his acquired neuromuscular weakness. However, there is not enough evidence regarding its effects in COVID-19 patients.

\section{ConcLusion}

Acquired neuromuscular weaknesses has been infrequently detected in patients with acute respiratory distress syndrome due to infection with SARS-CoV-2. In this case, the use of hydroxyzine, a drug with little or no known respiratory effects, triggered the presentation of weakness with acute hypercapneic respiratory failure. More studies to determine the respiratory effects of this drug and to characterize neuromuscular disorders in COVID-19 are needed.

Article citation: Bahi M, Aissaoui Y, Belhadj A, Qamouss Y, Seddiki R. Hydroxyzine revealing acquired neuromuscular weakness in a patient with COVID-19 disease. The Southwest Respiratory and Critical Care Chronicles 2021;9(40):74-76

From: Anesthesiology and Intensive Care Unit, Avicenne Military Hospital, Cadi Ayyad University, Marrakesh, Morocco

Submitted: $5 / 15 / 2021$

Accepted: 6/22/2021

Reviewer: Kenneth Nugent MD

Conflicts of interest: none

This work is licensed under a Creative Commons Attribution-Share A like 4.0 International License.

\section{REFERENCES}

1. Mao L, Wang M, Chen S, et al. Neurological manifestations of hospitalized patients with COVID-19 in Wuhan, China: A retrospective case series study. MedRxiv; 2020.

2. Ghannam M, Alshaer O, Al-Chalabi M, et al. Neurological involvement of coronavirus disease 2019: a systematic review. J Neurol 2020 Jun 19:1-19

3. Algahtani H, Subahi A, Shirah B. Neurological complications of Middle East respiratory syndrome coronavirus: a report of two cases and review of the literature. Case Rep Neurol Med 2016;2016:3502683.

4. Torbic H, Duggal A. Neuromuscular blocking agents for acute respiratory distress syndrome. J Crit Care 2019;49:179-184.

5. Doughty CT, Amato AA. Toxic myopathies. Continuum 2019; 25:1712-1731.

6. Varan O, Kucuk H, Tufan A. Myasthenia gravis due to hydroxychloroquine. Reumatismo 2015;67:849.

7. Gummi RR, Kukulka NA, Deroche CB, et al. Factors associated with acute exacerbations of myasthenia gravis. Muscle Nerve 2019;60:693-699.

8. Lauria JI, Marakello R, King BD. Circulatory and respiratory effects of hydroxyzine in volunteers and geriatric patients. Anesthesia and Analgesia: Current Researches 1968;47:378. 